\title{
Evaluation of water washing efficiency and erosion risk in an axial compressor for different water injection conditions
}

\author{
Giuliano Agati ${ }^{1, *}$, Francesca Di Gruttola ${ }^{1}$, Serena Gabriele $^{2}$, Domenico Simone ${ }^{3}$, Paolo \\ Venturini $^{1}$, Domenico Borello ${ }^{1}$ \\ ${ }^{1}$ Sapienza Università di Roma, Dipartimento di Ingegneria Meccanica e Aerospaziale, via Eudossiana \\ 18, 0084, Roma (Italy) \\ ${ }^{2}$ Baker Hughes, Viale F. Matteucci, 2, Florence, Italy \\ ${ }^{3}$ Aerospace Engineering, University of Brasilia (UnB) at Gama, 72444-240 Brasilia, DF, Brazil
}

\begin{abstract}
Gas turbines performance losses are mainly due to the deposition of dirt on the compressor blades that needs to be periodically removed. This is the reason motivating the presence of water washing systems (WWS) in most of the compressor gas turbines. Water washing is generally achieved by installing a number of nozzles on the compressor casing and spraying water that clean the dirty surfaces of the compressor. The side effect of such a technique is the rising risk of erosion due to the impact of water droplets on the compressor blades which is even more pronounced when dealing with online water washing systems that is done while the unit is at normal load. The design of these systems must balance benefits and disadvantages associated to the process itself. The benefits can be measured in terms of water washing efficiency that is a quantity not uniquely defined. In previous works, the authors introduced some indices useful to evaluate the spatial cleaning coverage (the wet to the total surface) and the quantity of water mass actually impacting the dirty surfaces (the impacted to injected mass). On the other hand, water washing erosion is a complex phenomenon depending on several parameters, such as the mechanical properties of the blade material, the impact velocity and angle and the droplet diameter. For this reason, the WWS are strongly influenced by the adopted nozzles and by the injection conditions. The present paper aims at assessing water washing for six different injection conditions in the first stage of a real axial compressor. Two-phase CFD simulations are carried out with Ansys Fluent where a User Defined Function implemented by the authors is used to properly model water droplet erosion mechanism and to obtain all the quantities needed to evaluate the washing quality. Results confirm the strong influence of the injection conditions on the main features of the washing system. The study is part of an ongoing partnership between Baker Hughes and Sapienza University of Rome aiming at maximizing the washing of the compressor blades while maintaining the erosion under specific thresholds.
\end{abstract}

\footnotetext{
* Corresponding author:giuliano.agati@uniroma1.it
} 


\section{Introduction}

As reported in the open literature, the loss of performance in a gas turbine engine is essentially related to compressor problems [1,2]. Among other issues, the deposition of particle entrained by the inlet air on the compressor blades alters the flow passage geometry provoking compressor derating. The operating conditions of the machine affects the particle deposition phenomenon [3-6]. According to Meher-Homji et al. [1] and Brooks [5], losses due to particle depositions are classified as recoverable, since they can be recovered during the engine operations without any parts replacement. Compressor washing is the technique used to reduce fouling related aerodynamic efficiency losses, and it can be performed both off line and online. Online washing in recent years has attracted great interest due to its ability to reduce compressor losses between two offline washes, thus reducing machine downtime and minimizing the impact on plant operation. Recent experiments [8] demonstrated that increasing the water-to-air ratio results in a higher washing efficiency and a slower performance degradation of the compressor. However, during online water washing the engine is working at (or close to) its nominal speed [9], hence water droplets hits the compressor blades at very high speed; this, in turn, potentially leads to the erosion of the blades [10,11]. There is therefore the need to satisfy two fundamental requests: on the one hand, we want to minimize the amount of water sprayed to minimize the risk of erosion, which is a non-recoverable damage $[1,5]$; on the other hand, the injected water amount must be enough to wash the blades.

To satisfy these requests the injection system plays a paramount role. According to their pressure, the injection systems are classified as low ( $\mathrm{p}<10$ bar) and high ( $\mathrm{p}>50$ bar) pressure [12]. The injection pressure affects the spray diameter, the cone angle and the injection velocity; all these parameters, together with the number and position of the injectors and the injection time, are linked to the water distribution in the machine, to the washing efficiency and to the risk of causing erosion on the blades [13-18]. Varying and optimizing these quantities result in a more or less efficient washing system, which in turn results in energy consumption, emission variation and duration of the blade life.

In some previous works, the authors presented a new water droplet erosion model specifically developed for erosion prediction of stainless steel compressor blades [19,20]. In [21], [22] and [23] the authors applied the model to predict water droplet erosion on different blade geometries and develop some indices with the aim of quantitatively evaluate the washing efficiency, a quantity that is not easy to evaluate since it depends on several specific operating conditions.

In the present paper, we analyse the effect of the water injection rate on the washing efficiency and the erosion in a first stage rotor of a compressor for a gas turbine engine. After a description of the models and computational approach used, we give the main numerical details related to the analysed geometry and the performed simulations. Then we present and discuss the results, in terms of erosion and washing efficiency indices. Some closing remarks will conclude the paper.

\section{Models and computational approach}

In the present study a real compressor is reproduced from the inlet case to the first-stage rotor, thus comprising the inlet region, casing, struts, inlet guide vanes (IGVs) and the first rotor. Simulations are performed by using Ansys Fluent 2020R2 [24]. The multi-phase flow solution was based on a Eulerian-Lagrangian one-way coupling approach. The turbulent field is modelled by using Unsteady (U)-RANS adopting the well-established, widely-accepted $k-\varepsilon$ realizable model. Standard wall-function was used for the near-wall treatment. The SIMPLEC scheme was selected for the pressure-velocity coupling. Spatial discretization for the 
pressure term was achieved by a second order upwind scheme while for all the other variables a first order scheme is adopted to increase the solution stability.

In all the simulated configurations, before injecting droplets a rotating flow field is solved until a converged solution is reached (about 4000 time steps) by imposing the maximum accepted residual down to $1.0 \mathrm{E}-05$ for the continuity equation. The converged fluid flow solution has stable residuals of about 5E-6 and 5E-5 for the turbulent kinetic energy (k) and for the turbulent dissipation rate $(\varepsilon)$, respectively. Once the fluid flow has reached convergence, transient simulations are run and the injected droplets are tracked in a Lagrangian framework until all of them exit the domain or impact one of the solid surfaces. In the transient simulations a time step $d t=3.0 \mathrm{E}-05$ is adopted for the fluid flow while the integration of droplets motion is achieved by an automated tracking scheme that permits to switch between a numerically stable implicit lower order scheme and a trapezoidal higher order one. Moreover, the accuracy control option permits the solution of droplets motion equation to be within a tolerance of $1.0 \mathrm{E}-05$.

To account for rotor blades rotation, the Multiple Reference Frame Model (i.e., frozen rotor) is adopted. Hence, the rotor cell zone mesh is fixed but the fluid flow equations are solved in the relative reference frame [24] (the angular velocity $\omega$ is equal to $7800 \mathrm{rpm}$ with respect to the negative $\mathrm{x}$-axis).

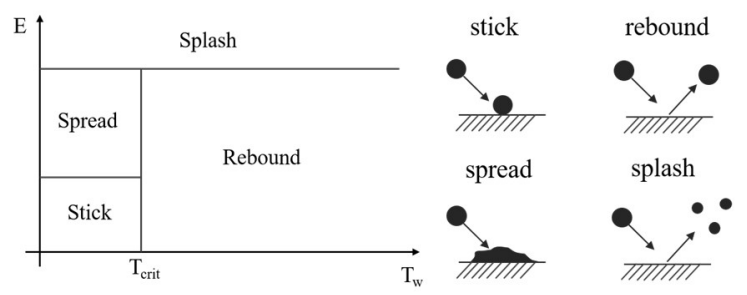

Fig. 1. Droplet impact regimes according to the Stanton-Rutland model [25, 21].

Droplets are injected by adopting the solid cone injection model available in Fluent [24], and they are tracked by adopting a Random Walk Model to account for the turbulence effect on their dispersion. The characteristic lifetime of the eddy $\left(\tau_{\mathrm{e}}\right)$ that needs to be defined in the Random Walk Model was calculated as a function of the fluid Lagrangian integral time $\mathrm{T}_{\mathrm{L}}$ [24] and ends up to be around 1.8E-04. Once a droplet hits a wall, the Stanton-Rutland model [25] is adopted to compute whether it sticks, spreads, splashes or rebounds, according to the local wall temperature and the impact energy (Fig. 1). In the present study, the wall temperature is always smaller than $T_{\text {crit }}$, thus droplets can only stick, spread or splash according to their impact energy. The splashing criteria are those defined in [26]. If a droplet splashes, secondary smaller droplets are generated, being their diameters sampled by a cumulative probability Weibull distribution function that was fitted to the experimental data from Mundo et al. [27]. Beside the impact model just introduced, at each droplet impact the User Defined Function (UDF) defined in [20] and implemented in Fluent, activates giving access to several droplet information useful to study the water droplet erosion process and the water washing efficiency. 


\section{Computational details}

\subsection{Domain and mesh}

The numerical domain with a close up of the rotor mesh are shown in Fig. 2. The air enters the domain from a rectangular opening at the top of the domain (blue region), and exits from the annulus section the $y z$ plane after the compressor rotor (green region). Water is injected in the domain through 14 injectors distributed along the red dotted line in Fig. 2-left, on the inlet case upstream of the struts. Water droplets mix with air along the passage, but the mixing is not uniform or homogeneous. Due to this non-uniformity, the non-commensurable ratio among struts, IGV and rotor blades as well as the geometry of rotor, it was not possible to assume periodicity or symmetry conditions. So the whole geometry has been simulated.

25.5 $\mathrm{M}$ tetrahedral cells are used to discretize the whole domain. Cells are clustered to the walls so that the first stripe of cells has $y+$ laying within the range 20-300, which is the interval of applicability of the wall functions.

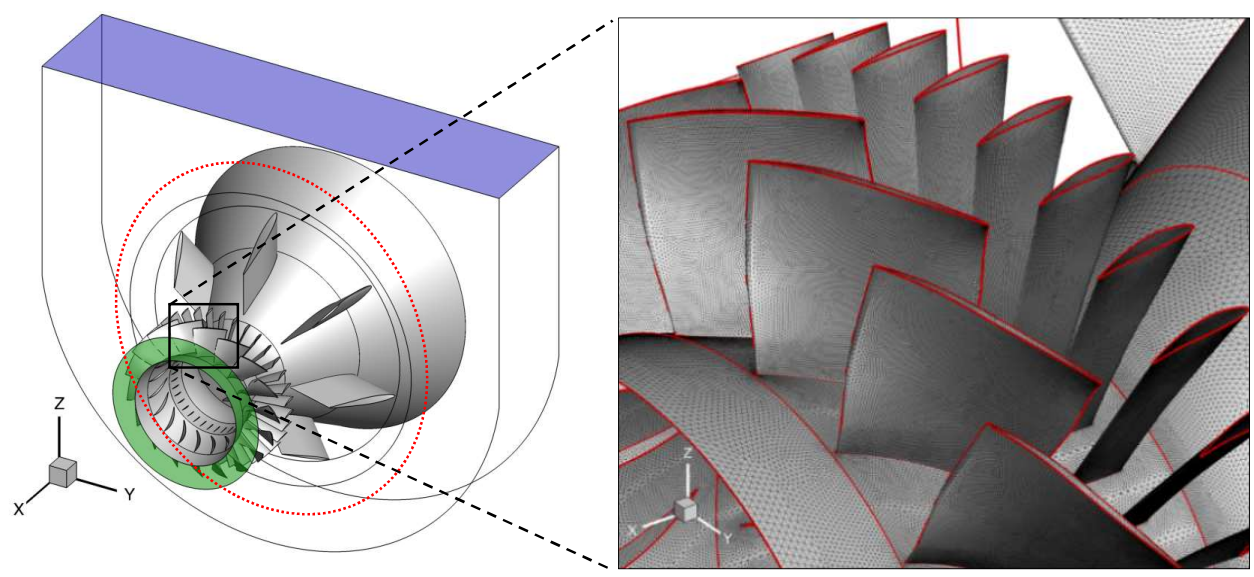

Fig. 2. Numerical domain (left) and zoom-in of the first-stage rotor (right); red dotted line: line of injector positions [21,23]. (c) 2021 Baker Hughes Company - All rights reserved.

\subsection{Boundary conditions}

At the inlet a mass flow inlet boundary condition (BC) is used, while a pressure outlet is used to fix the conditions at the outlet. The exit pressure is computed from the Eulerian work exerted by the rotor to the fluid, and compared with the industrial design value. The other surfaces are all treated as no slip stationary walls. Downstream the frozen rotor surface, noslip BCs are imposed on the rotor blades and hub walls in the relative frame of reference, while the casing was considered as a rotating wall with an angular velocity $\omega=7800 \mathrm{rpm}$. Water is injected by 14 injectors distributed along the red dotted line reported in Fig. 2-left. The cone angle and droplet size distribution vary according to the water flow rate (see Section 3.3 for details).

\subsection{Performed simulations}

In the present study we reproduce the online water washing process under different water flow rates. For data confidentiality reasons, the water mass flow used in the simulations is 
normalized (NWF); the whole range $(0.25-1.0)$ is covered using two kind of nozzles, namely $\mathrm{N} 1$ and $\mathrm{N} 2$, the former ranging between 0.25 and $0.50 \mathrm{NWF}$, the latter between 0.625 and 1.0. Table 1 reports the NWFs analysed in the present paper and the respective nozzle name, together with the injection angles and droplet size ranges. For each NWF the respective size range is divided into six classes, covering about $80 \%$ of the total injected mass, in order to have reliable results.

Table 1. Injector name, NWF, injection angle and droplet size range in the performed simulations.

\begin{tabular}{|c|c|c|c|}
\hline Nozzle & NWF & $\begin{array}{c}\text { Injection } \\
\text { angle }\left(^{\circ}\right)\end{array}$ & $\begin{array}{c}\text { Droplet size } \\
\text { range }(\boldsymbol{\mu m})\end{array}$ \\
\hline \multirow{3}{*}{ N1 } & 0.250 & 53.0 & $50-305$ \\
\cline { 2 - 4 } & 0.375 & 71.5 & $45-277$ \\
\cline { 2 - 4 } & 0.500 & 72.5 & $40-250$ \\
\hline \multirow{3}{*}{ N2 } & 0.625 & 84.5 & $35-305$ \\
\cline { 2 - 4 } & 0.750 & 84.5 & $35-281$ \\
\cline { 2 - 4 } & 1.000 & 87.5 & $35-242$ \\
\hline
\end{tabular}

\section{Results and Discussion}

In the following section we report the main results in terms of erosion damage and water washing efficiency. Since the flow field was analysed in previous publications (i.e., [21,22]), here we just provide the basics findings, just to frame the erosion results in a more clear and complete framework.

\subsection{Flow field}

At the inlet section flow field is symmetric with respect to the $z$ axis. Fig. 3-left shows the flow field streamlines in the absolute reference frame coloured with the velocity magnitude. In Fig. 3-right the streamlines are plotted in the relative reference frame. The work provided to the fluid through the rotor is responsible of a pressure increase rather than a flow acceleration.
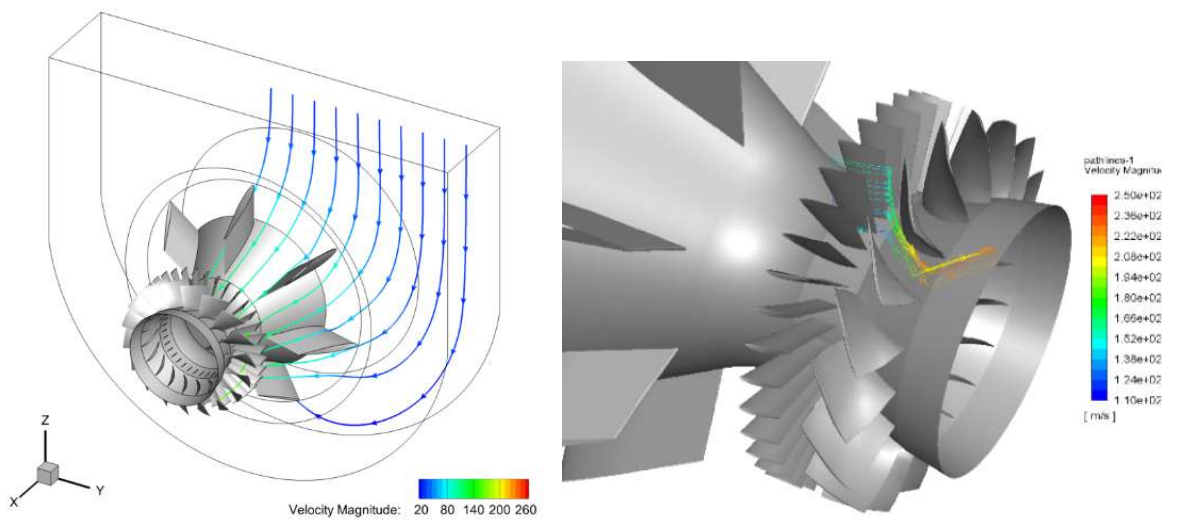

Fig. 3. Streamlines coloured with velocity magnitude in the whole domain (left) and a close-up of the rotor region [21]. (C) 2021 Baker Hughes Company - All rights reserved. 
An overall view of pressure and velocity fields within the domain are reported in Fig. 4. As shown, the inlet value of the pressure is similar to the exit one, reaching its maximum at the exit of the rotor, at the tip of the rotor blades. In Fig. 4-c) a close-up of the rotor region is reported, showing the contour plots of velocity magnitude on different streamwise surfaces representing the IGV inlet as well as rotor inlet, middle and outlet sections. As expected, the velocity field follows the pressure variation imposed by the rotor action.
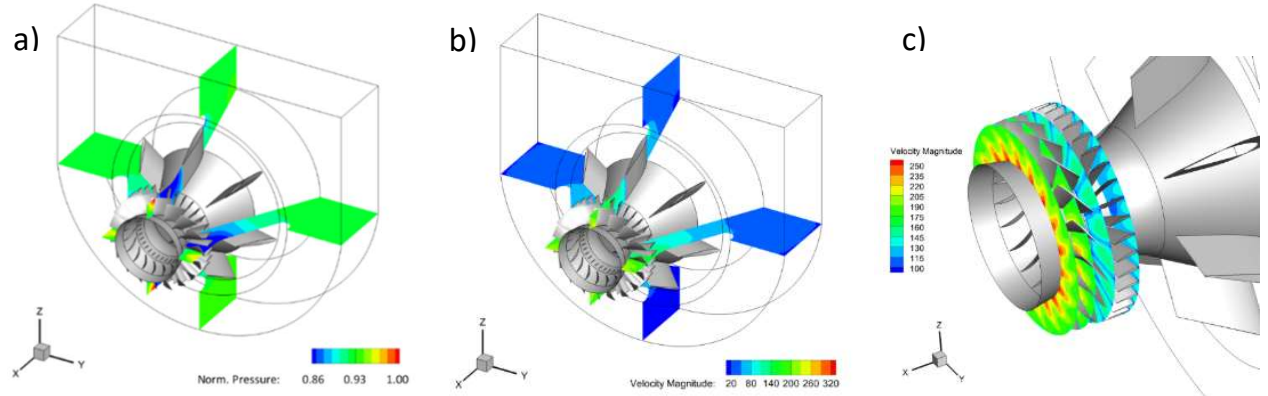

Fig. 4. Pressure and velocity magnitude contour plots on the middle $y$-and $z$ - sections ( $\mathrm{a}$ and $\mathrm{b}$, respectively), and a close-up of the rotor region ( $x$ - sections; c) [21]. (C) 2021 Baker Hughes Company - All rights reserved.

\subsection{Washing efficiency}

Since there are no standard quantities used to evaluate the washing efficiency of a compressor washing system, in [21-23] we introduced some indices that, together with other considerations, can help in the evaluation. Here we present these indices calculated in the new configurations listed in Table 1. For the sake of clarity, before discussing the results we briefly recall the definition of the water washing efficiency indices:

- $\quad$ WTS (Wet to Total Surface, \%): percentage of a surface hit by water droplets.

- ITM (local Impacted Mass to Total impacted water Mass, \%): percentage of the impacted mass hitting a specific surface with respect to the total impacted mass.

- IMWS (Impacted water Mass per unit of Wet Surface, -): ratio between impact water mass and wet surface. This quantity is normalized by its maximum so it varies in the range $0.0-1.0$.

In analysing the droplets impacts behaviour on the rotor, first of all it is important to point out that in the present work the frozen rotor approach was selected as the most appropriate to take into account the rotation of the rotor. In fact, the rotation speed of the rotor is really high (more than $7000 \mathrm{rpm}$ ), and considering a rotating mesh for the considered domain would require enormous computational time and resources. By adopting the frozen rotor approach, droplets end up to impact not evenly the rotor blades as it should be. For this reason, the washing indices will be analysed on every single blade but also their maximum values will be reported. These values can be assumed to be representative of all the rotor blades.

Fig. 5 reports WTS and ITM indices for the machine regions (namely Inlet case, Struts \& Cone, IGV and Rotor) composing the considered domain. It is evident (Fig. 5-top) that the region showing the most variable WTS index with NWF is the Inlet case. It shows a continuous increase of that index as the NWF increases, starting with WTS about $15 \%$ in the case of $\mathrm{NWF}=0.25$ and reaching more than $55 \%$ in the case of $\mathrm{NWF}=1.0$. WTS variations in the other regions are less pronounced.

The rotor region in particular, shows an asymptotic trend: using a NWF $>0.625$ does not provoke any relevant variation in the wet-surface index, which stabilizes around $24 \%$. The situation is similar for ITM index (Fig. 5-bottom) but with some differences. The Inlet cone is 
still the region most sensitive to NWF variations, showing an increase of ITM index but in this case the variation is less evident (about 20\%). The ITM in the Struts\&Cone region, on the contrary, decreases with the increase of NWF, going from about $39 \%$ for NWF $=0.25$ to about $28 \%$ for the case at NWF=1.0. This can be related to what happens in the Inlet case region: the larger mass hitting the Inlet region observed by increasing the NWF results in a minor number of droplets' impacts on the rest of the domain. In the rotor region a decreasing asymptotic trend of the ITM index with NWF is detected: by injecting with a NWF $>0.5$ the ITM index stabilizes around a value of $25 \%$.

The normalized impacting water mass per unit surface (IMWS) is reported in Fig. 6. In all the domain regions there is an increase of IMWS with NWF. This could appear to be in contrast with the ITM trend. However, ITM represents the percentage of the impacted water hitting a specific surface with respect to the whole impacted mass, but it is not related to the amount of water actually impacting a surface. Increasing the water mass hitting a unit surface on one hand could result in a larger washing efficiency, but on the other hand it increases the risk of erosion. Within this framework, in the present geometry it is clear that IGV and Rotor regions are the most exposed to erosion risk.

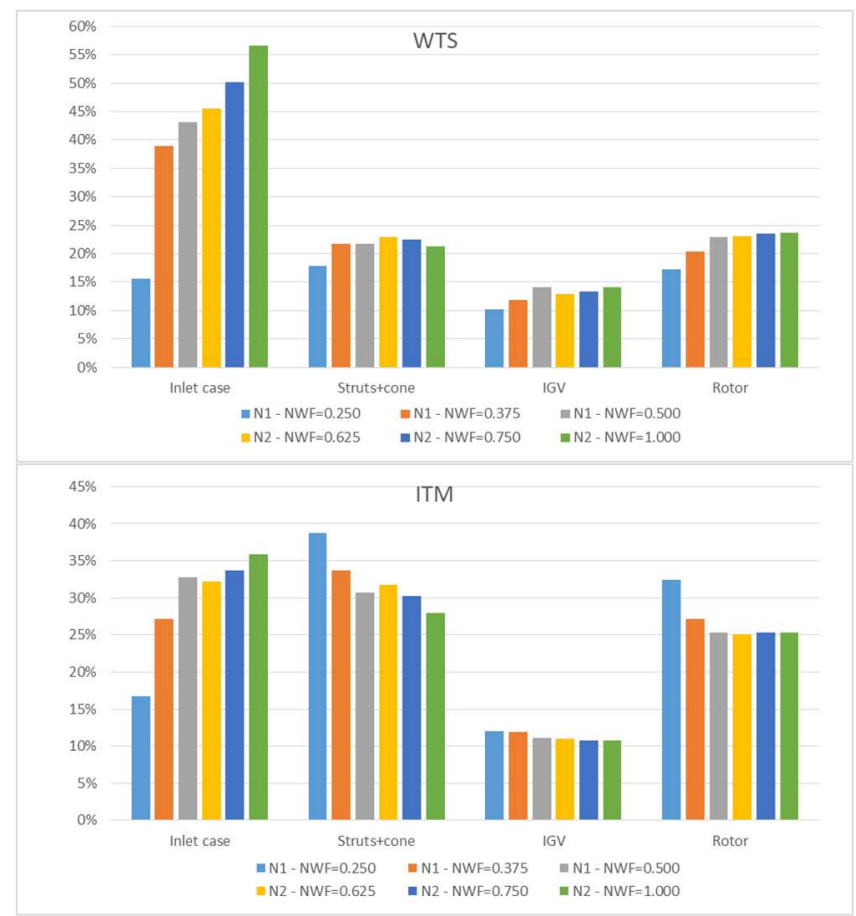

Fig. 5. WTS (top) and ITM (bottom) in the domain regions.

Since the most mechanically stressed and critical part of the machine is the rotor, in the following we focus the analysis on that region. Fig. 7 recalls the injectors and blades names.

Fig. 8 reports the WTS index of the rotor blades as a function of NWF. When the nozzle N1 is used (Fig. 8-left) the rotor is much more sensitive to the injection conditions variations. Indeed, apart from some blades in the bottom part of the rotor (rot09-rot14) showing small WTS variations (maximum 5.0\%) with NWF, the other blades (rot01-rot08 and rot15-rot18) show more pronounced differences. 


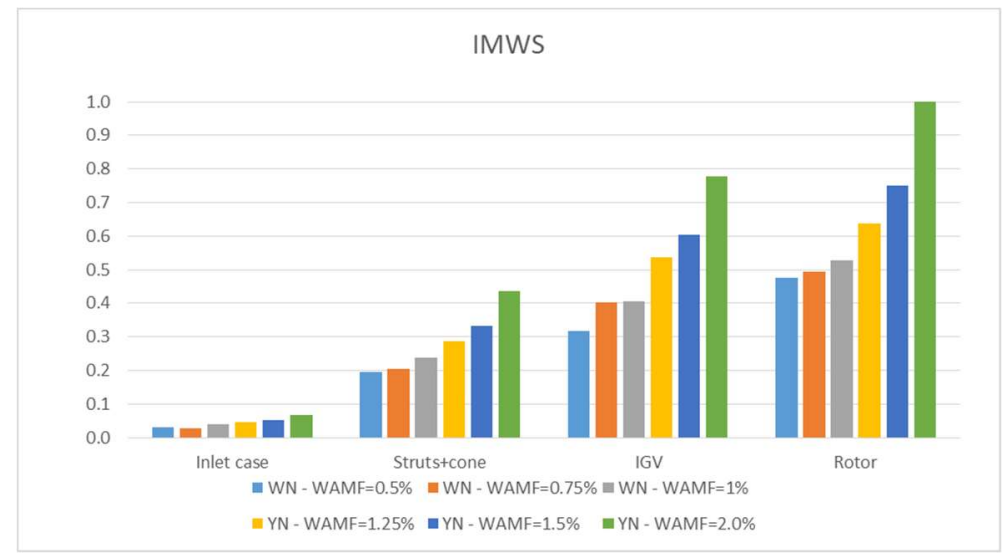

Fig. 6. IMWS in the domain regions.

This is due to the droplet dynamics in the considered configuration: most of the droplets injected are dragged toward the bottom part of the inlet case where much of them are trapped; the few rest impact the top part of the inlet cone, eventually splash and then continue their path toward the rotor. Increasing the water flow rate results in a major number of splashed droplets on the top part of the cone (since the impact energy will be higher), leading to an increase of small droplets impacting the rotor blades. WTS in the top rotor region, therefore, sensitively increases with NWF. On the contrary, droplets concentrating in the bottom part of the casing undergoes less splashes, which means that their dynamic (and hence the WTS index for the bottom rotor blades) is less affected by the increase of the water flow rate. The relative velocity of the rotor makes the WTS distribution make asymmetrical along the rotor blades. To further increase the water mass flow rate, nozzle N2 should be adopted. As said, $\mathrm{N} 2$ presents a different injection cone, inlet velocity and droplets size distribution (resulting in a globally larger droplet inertia), and this leads to a less variable WTS behaviour (Fig. 8right).

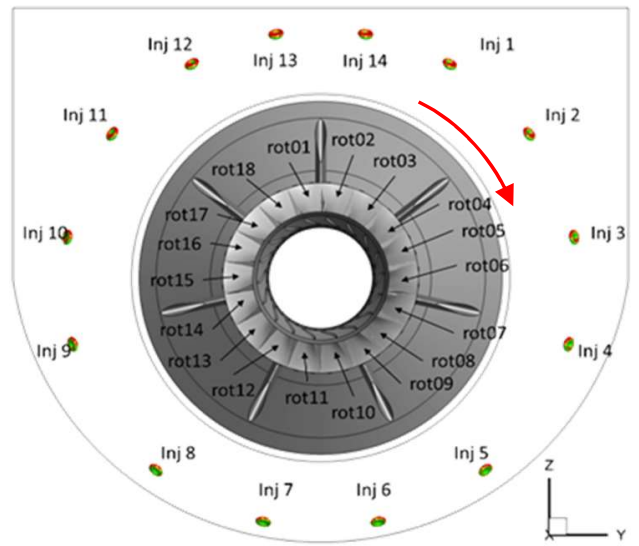

Fig. 7. Reference names and potions of injectors and rotor blades [23]. (C) 2021 Baker Hughes Company - All rights reserved. 


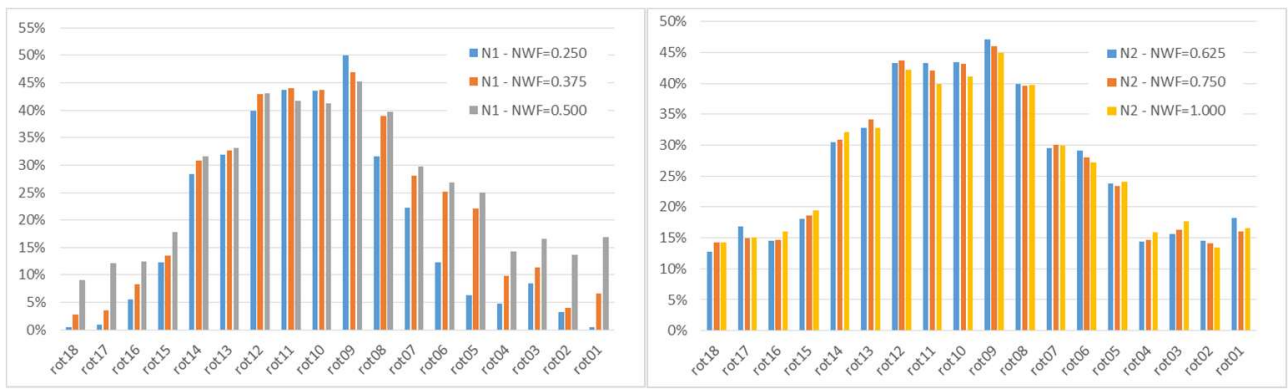

Fig. 8. WTS of the rotor blades as a function of NWF. Left: nozzle N1; right: nozzle N2.

As previously mentioned, the different WTS values along the rotor blades are due to the frozen rotor approach adopted for the present case. It follows that in real compressors, all rotor blades will have the same wetted surface, here assumed to be the maximum predicted by the simulations. Fig. 9 shows the maximum WTS value as a function of NWF, predicted at blade rot09. What comes out from the figure is that the maximum of WTS decreases as NWF increases, even if passing from nozzle N1 to nozzle N2 the variation range becomes less pronounced (from about $5 \%$ for $\mathrm{N} 1$ to about $2 \%$ for N2). This result seems in contradiction with what seen in Fig. 5-top. However, Fig. 5-top represents the whole rotor wet surface computed as sum of the wet surfaces of each blade, not considering that for some of the blades the wet surface could be the same.

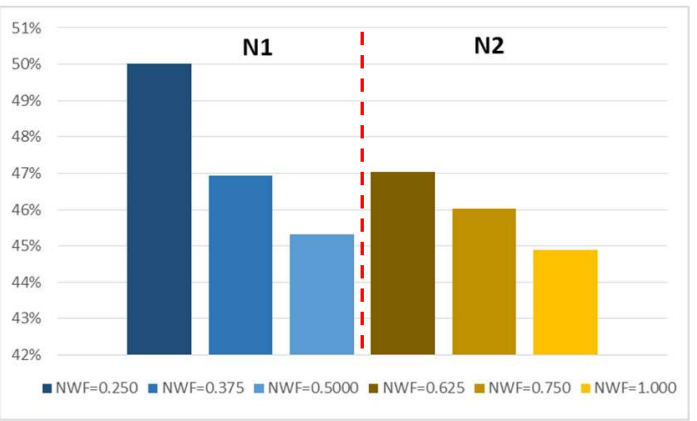

Fig. 9. Maximum of WTS on rot09 as a function of NWF. Blue-scale bars: nozzle N1; Yellow-scale bars: nozzle N2.

Fig. 10 reports ITM and IMWS on the rotor blades for nozzles N1 an N2. ITM for nozzle N2 does not vary significantly as the NWF increases. On the contrary, N1 nozzle shows some more evident variations, especially for blades rot11-rot14 and rot08. IMWS variations are evident in both the nozzles: in N1 nozzle, variations are concentrated on blades rot08-rot10, showing a strong increase of the IMWS index with NWF. Using N2 nozzle results in some slightly larger variations in a wider range of blades (namely rot08-rot12).

Referring to Fig. 8 and Fig. 10, it is worth noticing that for all the NWF values, maximum of WTS is predicted on blade rot09, while maximum of ITM and IMWS are always on rot10 (a part from $\mathrm{NWF}=0.250$ ). As a general trend, in the present configuration the bottom part of the rotor is the most critical from the erosion risk point of view but also the mostly washed. 


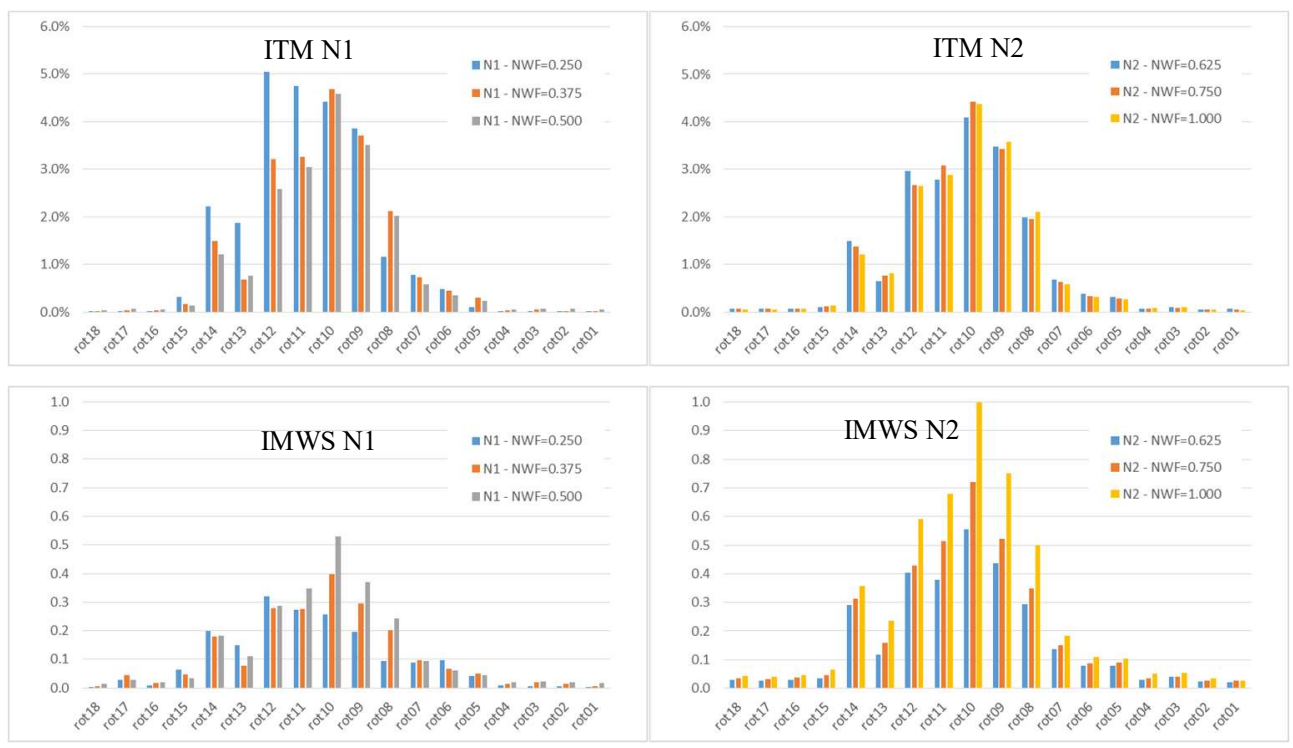

Fig. 10. ITM (top) and IMWS (bottom) of the rotor blades as a function of NWF. Left: nozzle N1; right: nozzle N2.

Fig. 11 shows the variation of ITM and of IMWS on rot10 by varying the NWF. ITM index (Fig. 11-left) shows a very weak decrease as NWF increases, so that it can be considered constant with NWF. On the contrary, IMWS shows a larger variation and a clear trend, continuously increasing with NWF from 0.3 to 1.0 .
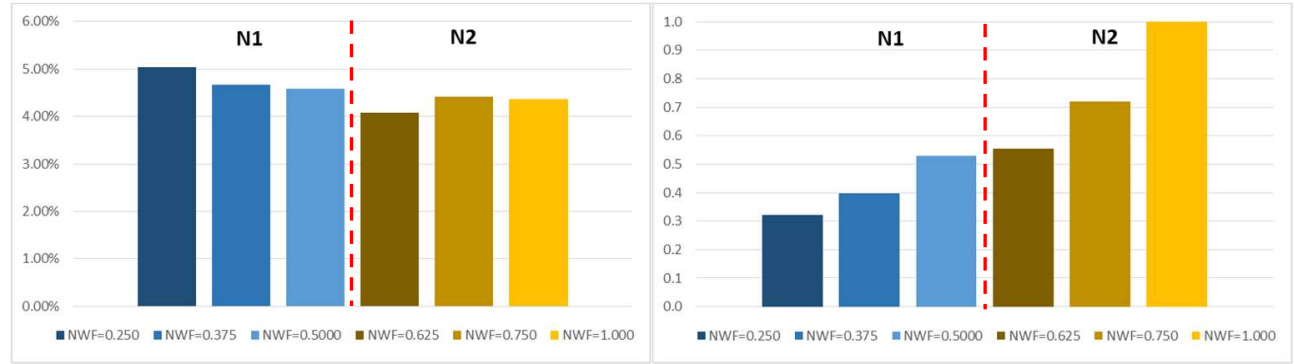

Fig. 11. Maximum of ITM (left) and IMWS (right) on rot10 as a function of NWF. Blue-scale bars: nozzle N1; Yellow-scale bars: nozzle N2.

\subsection{Erosion prediction}

First of all, it is important to recall that also the erosion prediction is affected by the frozen rotor approach here adopted. Erosion is not evenly distributed along rotor blades as it will result in the real configuration. For this reason, the erosion values should be divided by the number of rotor blades, since, in the real application, the mass impacting one rotor should be spread over all the rotor blades. However, the results here reported are still interesting since they show the regions of the blades affected by erosion phenomena. Moreover, erosion variations by injecting with different NWFs can be considered valid once the assumptions made are clear. In Fig. 12 and in Fig. 13 the erosion profiles on the rotor are shown for blades pressure and suction sides, respectively. For both the sides of the blades, more evident differences can be observed in the erosion profiles when increasing the NWF in the N1 nozzle. 

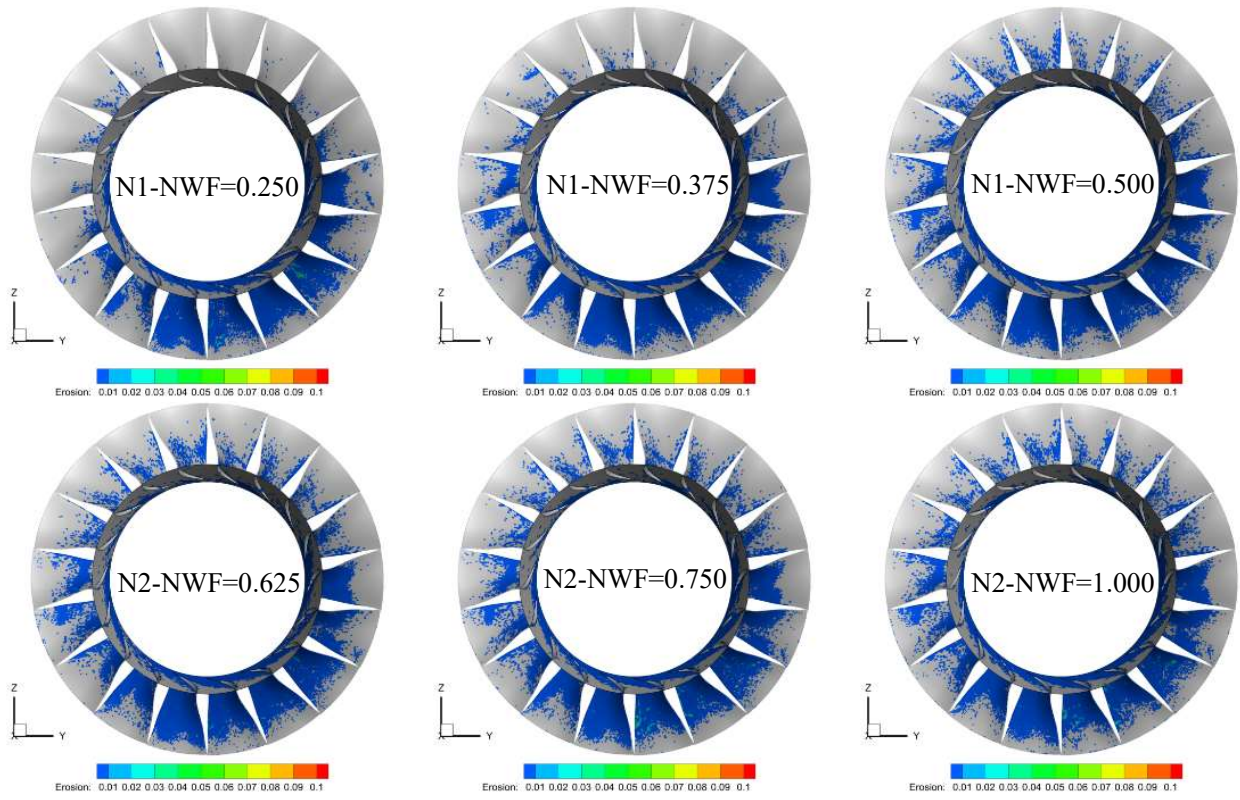

Fig. 12: Erosion profiles predicted on the rotor blades pressure sides for the set of simulations here analysed. The values of erosion were normalized by the maximum recorded for every case but the legend contour varies between 0 and 0.1 to make the levels differences more evident. (C) 2021 Baker Hughes Company - All rights reserved.
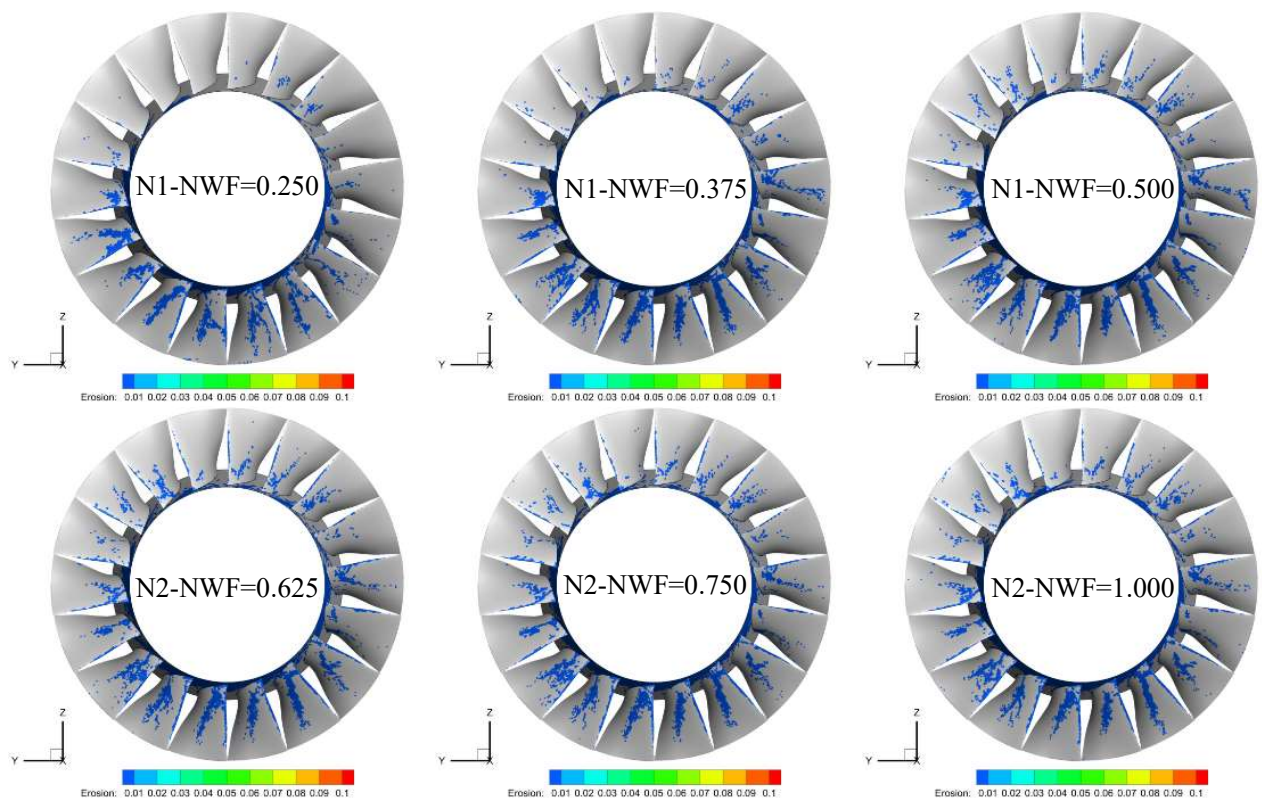

Fig. 13: Erosion profiles predicted on the rotor blades suction sides for the set of simulations here analysed. The same procedure to normalize the erosion explained in Fig. 12 is here adopted. (C) 2021 Baker Hughes Company - All rights reserved.

Focusing on the erosion profiles reported on the pressure sides (Fig. 12), the low-right blades (from rot06 to rot12) present very similar erosion distributions from a qualitative point of 
view. All the other blades show always larger eroded regions on the pressure side by increasing the NWF. It is worth noticing that, for the set of simulations here analysed, the tip of the blades remains not eroded since most of the droplets impacts occur in the lower part of the blades. The blades suction sides (Fig. 13) are in general less affected than the pressure sides. This is also due to the rotational effect, which pushes the droplets entering the rotor towards the blades pressure sides. Only the droplets with higher inertia will maintain their relative motion towards the blades suction sides. On these blade sides, some strips in the erosion contour are evident for the whole set of simulations here assessed. This might be due to the particular position coupling between the IGVs and the rotor blades. In most of the configurations analysed in the present paper, the rot09 is found to be the blade where the erosion peak was detected. For this reason, in Fig. 14, the erosion profiles on the pressure sides of this blade are shown by varying the NWF.

Most of the rot09 pressure side is subject to erosion for all the NWF here assessed. For the last two simulations $(\mathrm{NWF}=0.750$ and $\mathrm{NWF}=1.000)$, more pronounced material removal is observed around the blade mid-span region. In Fig. 14, the erosion contour has been normalized by the maximum erosion value found in the whole domain of each simulation.

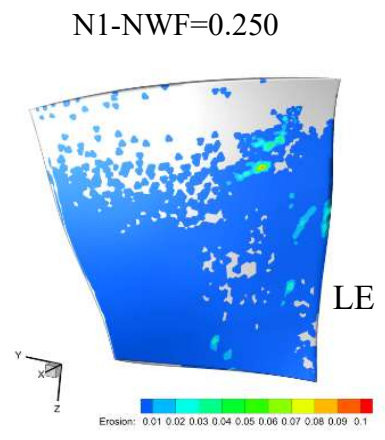

$\mathrm{N} 2-\mathrm{NWF}=0.625$

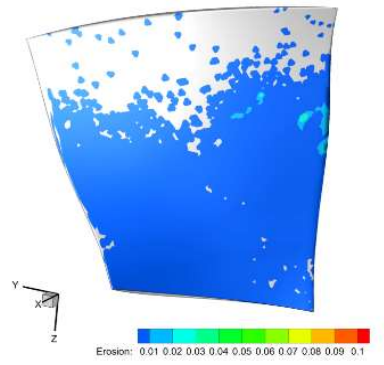

$\mathrm{N} 1-\mathrm{NWF}=0.375$

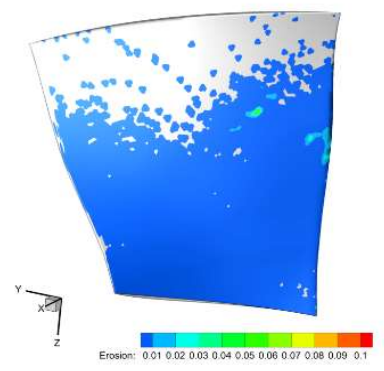

$\mathrm{N} 2-\mathrm{NWF}=0.750$

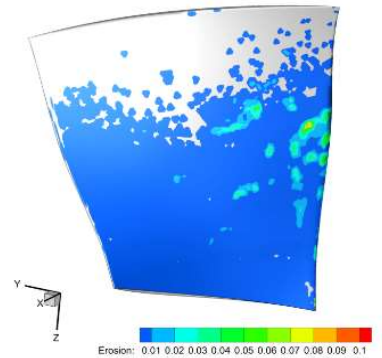

$\mathrm{N} 1-\mathrm{NWF}=0.500$

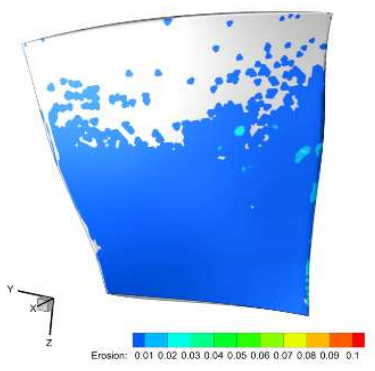

$\mathrm{N} 2-\mathrm{NWF}=1.000$

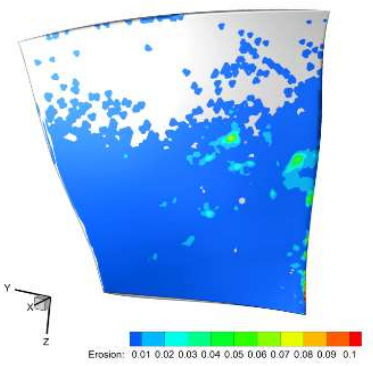

Fig. 14: Erosion profiles predicted on rot 08 pressure sides for the set of simulations here analysed. Also here the erosion values were normalized as explained in Fig. 12. (C) 2021 Baker Hughes Company - All rights reserved.

Hence, not quantitative comparison can be made by watching uniquely at the contour plots. For this reason, in Table 2 the values of the erosion peaks detected for each configuration is reported. The values have been normalized with respect to the maximum of erosion found in the whole set of simulation that resulted to be, as expected, in the case where the larger quantity of mass was injected, i.e. $\mathrm{NWF}=1.000$. This is the worst configuration from the erosion risk point of view. 
By recalling Table 1 where the main features of each injection were summarized, one could point out that the flow rate per nozzle and the injection velocity (which are the highest in the configuration at $\mathrm{NWF}=1.000$ ) play a major role in the erosion phenomenon in comparison with the spray angle and the droplets size. In fact, generally speaking, the larger the spray angle, the higher will be the dispersion of the injected droplets that will impact in wider regions, resulting in an erosion more widespread but with lower peaks. Indeed, for each nozzle by increasing the NWF, the larger droplets dimension diminishes which should result in less erosive impacts. Nevertheless, Table 2 suggests that the erosion peak increases with the NWF. It is also worth observing that the trend of the erosion peaks with the NWF is similar to the IMWS index reported in (Fig. 11-right) which is confirmed to be a useful parameter to evaluate the erosion risk associated to water washing systems.

Table 2: Erosion peak normalized by the global maximum that was reached in all the simulations. The highest erosion was found for the case $\mathrm{N} 2$ at $\mathrm{NWF}=1.0$ was used.

\begin{tabular}{|c|c|c|c|c|c|c|}
\hline Nozzle & \multicolumn{3}{|c|}{$\mathrm{N} 1$} & \multicolumn{3}{c|}{$\mathrm{N} 2$} \\
\hline NWF & 0.250 & 0.375 & 0.500 & 0.625 & 0.750 & 1.000 \\
\hline Erosion peak & 0.28 & 0.36 & 0.38 & 0.51 & 0.75 & 1.00 \\
\hline
\end{tabular}

However, the presence of an erosion peak on a blade does not mean that it is the most eroded. Considering the volume of removed material (Fig. 15), computed as integral over the blade surface of the erosion thickness, it comes out that rot10 (and not rot09) is the one having a larger material removal for all the NWFs apart from $\mathrm{NWF}=0.250$. In this latter case, the most eroded blade is rot09. This is a further confirmation of the capability of the considered indices to predict the erosion risk due to a given configuration.

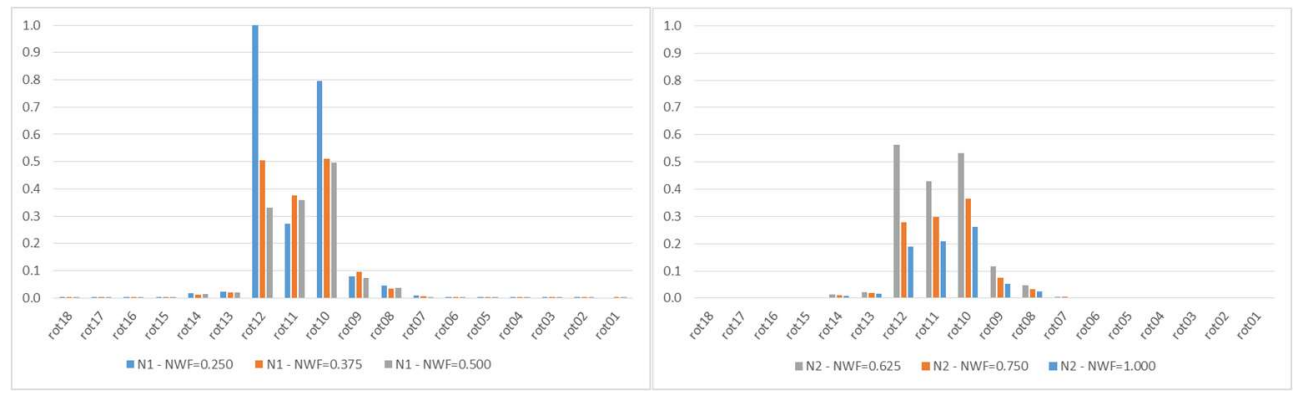

Fig. 15. Normalized volume removed from the blades as a function of NWF. Nozzle N1: left; nozzle N2: right.

\section{Conclusions}

In the present paper we studied the effect of water flow rate on erosion damage and on water washing efficiency on the first-stage rotor of an axial compressor. A series of CFD simulations have been performed to better understand the complex phenomenon of the online water washing in a real full-rotor compressor geometry by varying the injection conditions. The 3D unsteady simulations were carried out by using Ansys Fluent, where the two-phase flow solution was based on a Eulerian-Lagrangian approach. The erosion phenomenon was completely handled by the authors through the development of a specific semi-empirical erosion model implemented in the solver through the use of a User Defined Function.

In past works some indices were developed by the authors to evaluate the quality and the erosion risk associated to online water washing. Here, these parameters were extensively 
used to study the effect of different injection configurations. The main outcomes of the analysis are reported below.

- $\quad$ By increasing the nozzle flow rate (NWF in the text) the wet surface (WTS) always increases at the inlet of the domain while in the rotor this parameter shows an asymptotic trend: using a NWF $>0.625$ does not provoke any relevant variation in the wet-surface index. By focusing on the impacted mass, in the rotor region a decreasing asymptotic trend of the ITM index with NWF is detected: by injecting with a NWF $>0.5$ the ITM index stabilizes around a value of $25 \%$. On the other side, on the rotor blades the normalized impacting water mass per unit surface (IMWS) constantly increases with NWF.

- Different patterns of wet surface are found for every single blade because of the use of the frozen rotor approach. The lower blades are in general the most affected by droplets impact but when using nozzle N1 the rotor is much more sensitive to the injection conditions variations which are found mainly on the top blades. These variations are associated to the splashing phenomenon which mainly occurs on the top part of the internal cone.

- When concentrating to the maximum WTS detected between the rotor blades (which is assumed to reproduce all the rotor blades behaviour in the real rotating situation) the WTS decreases as NWF increases. Even if the overall WTS increases along the full rotor surface, the larger spread of the water results in a lower WTS maximum.

- All the blades but the low-right ones (from rot06 to rot12), show always larger eroded regions on the pressure side by increasing the NWF. Here the tip of the blades remains not eroded since most of the droplets impacts occur in the lower part of the blades. The blades suction sides are in general less affected by droplets hits and by erosion phenomena.

- The normalized values of the erosion peaks are reported for all the simulations performed. The case where the larger quantity of mass was injected, i.e. $\mathrm{NWF}=1.000$. was found to be the worst configuration from the erosion risk point of view. The flow rate per nozzle and the injection velocity seem to play a major role in the erosion phenomenon in comparison with the spray angle and the droplets size.

- The increasing trend of the erosion peaks with the NWF is similar to the IMWS index which is confirmed to be a useful parameter to evaluate the erosion risk associated to water washing systems.

In conclusion, we summarized in Table 3 the correlations between the indices considered and the main erosion-related quantities. It is evident that the proposed indices are able to predict the erosion risk of different water washing configurations. In particular, the WTS index and the Erosion peak seem to have a correlation that will be further explored in the future.

Table 3: Rotor blades containing the maximum values of the water washing efficiency indices and the main erosion quantities as a function of the NWF.

\begin{tabular}{|c|c|c|c|c|c|c|}
\hline Nozzle & NWF & WTS & ITM & IMWS & $\begin{array}{c}\text { Erosion } \\
\text { peak }\end{array}$ & $\begin{array}{c}\text { Eroded } \\
\text { volume }\end{array}$ \\
\hline \multirow{3}{*}{ N1 } & 0.250 & Rot09 & Rot12 & Rot12 & Rot08 & Rot12 \\
\cline { 2 - 7 } & 0.375 & Rot09 & Rot10 & Rot10 & Rot09 & Rot10 \\
\cline { 2 - 7 } & 0.500 & Rot09 & Rot10 & Rot10 & Rot09 & Rot10 \\
\hline \multirow{3}{*}{ N2 } & 0.625 & Rot09 & Rot10 & Rot10 & Rot09 & Rot12 \\
\cline { 2 - 7 } & 0.750 & Rot09 & Rot10 & Rot10 & Rot08 & Rot10 \\
\cline { 2 - 6 } & 1.000 & Rot09 & Rot10 & Rot10 & Rot09 & Rot10 \\
\hline
\end{tabular}


An even stronger correlation is found between the mass indices ITM-IMWS and the eroded volume. In this case, in fact, the blades containing the maximum values of ITM and IMWS are the same (apart from one) of those having the larger eroded volume. Therefore, the use of such indices can be considered as a valid tool not only to estimate the water washing efficiency but also to compare the erosion risk of different configurations.

\section{References}

1. C.B. Meher-Homji, M A. Chaker, H.M. Motiwala, Gas Turbine Performance Deterioration, Proceedings of 30th Turbomachinery Symposium, Houston, Texas (2001)

2. G.F. Aker, H.I.H. Saravanamuttoo, "Predicting gas turbine performance degradation due to compressor fouling using computer simulation technique", Journal of Engineering for Gas Turbines and Power 111, pp. 343-350 (1989)

3. G. Agati, D. Borello, F. Rispoli, A. Salvagni, P. Venturini, An innovative approach to model temperature influence on particle deposition in gas turbines, Proceedings of the ASME Turbo Expo (2016)

4. G. Agati, D. Borello, F. Rispoli, A. Salvagni, P. Venturini, "Numerical simulation of a particle-laden impinging jet: Effect of wall curvature on particle deposition", Proceedings of the ASME Turbo Expo (2017)

5. G. Agati, D. Borello, G. Camerlengo, F. Rispoli,, J. Sesterhenn, "Direct Numerical Simulation of an Oblique Jet in a Particle-Laden Crossflow", ERCOFTAC Series (2020)

6. G. Agati, D. Borello, G. Camerlengo, F. Rispoli,, J. Sesterhenn, "DNS of an Oblique Jet in a Particle-Laden Crossflow: Study of Solid Phase Preferential Concentration and Particle-Wall Interaction", Flow, Turbulence and Combustion (2020)

7. F.J. Brooks, "GE gas turbine performance characteristics", GE Power Systems, Report GER-3567H (2000)

8. S. Madsen, L.E. Bakken, 2018, "Gas turbine fouling off-shore; effective online water wash through high water-to-air ratio", Proceedings of the ASME Turbo Expo 2018, paper no. GT2018-75618 (2018)

9. H. Margolis, "U.S. Navy on-line compressor washing of marine gas turbine engines gas turbine engines", Proceedings of the International Gas turbine and Aeroengine Congress and Exposition, Oralndo, Florida, paper no. 91-GT-309 (1991)

10. M. Wall, R. Lee e S. Frost, "Offshore gas turbines (and major driven equipment) integrity and inspection guidance notes", Research Report 430 prepared by ESR Technology Ltd for the Health and Safety Executive (2006).

11. C. B. Meher-Homji e A. Bromley, "Gas Turbine Axial Compressor Fouling and Washing”, Proceedings of 33th Turbomachinery Symposium, Houston, Texas, pp. 163192 (2004)

12. E. Syverud, L. E. Bakken, “Online Water Wash Tests of GE J85-13”, Paper presented at the ASME Turbo Expo: Land, Sea and Air, Reno, Nevada, USA, Paper No. GT 2005$68702(2005)$

13. J. P. Stalder, J. Sire, "Salt Percolation Through Gas Tur-bine Air Filtration System and Its Contribution to Total Contaminant Level", Proceedings of the International Joint Power Generation Conference, New Orleans, Louisiana, Paper No. JPGC2001/PWR$19148(2001)$

14. F. C. Mund, P. Pilidis, "Online compressor washing: a numerical survey of influencing parameters", Proceedings of the Institution of Mechanical Engineers, p. 15 (2005) 
15. J.P. Stalder, P. van Oosten, "Compressor washing maintains plant performance and reduces cost of energy production", Proceedings of ASME International Gas Turbine \& Aeroengine Congress \& Exposition, Hague, Netherlands, Paper No. 94-GT-436 (1994)

16. P. McDermott, “Apparatus for cleaning gas turbine engine.” US Patent No. 5273395 (1993)

17. PNR. "Spray Engineering Handbook". Europe. Commercial Catalogues (2007)

18. E. Whaba e H. Nawar, "Multiphase flow modelling and optimization for online wash systems of gas turbines", Elsevier, pp. 7549-7560 (2013)

19. M. Andreoli, S. Gabriele, P. Venturini, D. Borello, "New model to predict water droplets erosion based on erosion test curves. Application to on-line water washing of a compressor", Turbo Expo 2019, paper no. GT2019-92033 (2019)

20. P. Venturini, M. Andreoli, D. Borello, F. Rispoli, S. Gabriele, "Modelling of water droplets erosion on a subsonic compressor cascade". Flow, Turbulence and Combustion, 103(4), pp.1109-1125 (2019)

21. G. Agati, F. Di Gruttola, S. Gabriele, D. Simone, P. Venturini, D. Borello, "Water washing of axial flow compressors: numerical study on the fate of injected droplets", ATI Virtual Conference, (2020)

22. F. Di Gruttola, G. Agati, P. Venturini, D. Borello, F. Rispoli, S. Gabriele, D. Simone, "Numerical study of erosion due to online water washing in axial flow compressors, Proceedings of ASME Turbo Expo 2020," Turbomachinery Technical Conference and Exposition, GT2020-14767 (2020)

23. F. Di Gruttola, G. Agati, P. Venturini, D. Borello, F. Rispoli, S. Gabriele, D. Simone, "Numerical study of droplet erosion in the first-stage rotor of an axial flow compressor", Turbo Expo 2021, paper no. GT2021-59661 (2021)

24. ANSYS Fluent Theory Guide, Release 2020R2. ANSYS, Inc., Southpointe (2018)

25. D.W. Stanton, C. J. Rutland, "Multi-Dimensional Modeling of Thin Liquid Films and Spray-Wall Interactions Resulting from Impinging Sprays", International Journal of Heat and Mass Transfer, 41, pp. 3037-3054 (1998)

26. A.L., Yarin, D.A. Weiss, "Impact of drops on solid surfaces: self-similar capillary waves, and splashing as a new type of kinematic discontinuity”, J Fluid Mech, 283, pp. 141-73 (1995)

27. C.M undo, M. Sommerfeld, C. Tropea, "Droplet-wall collisions: experimental studies of the deformation and breakup process", International Journal of Multiphase Flow, 21(2), pp.151-173 (1995) 